\title{
Augmenting matrix factorization technique with the combination of tags and genres
}

\author{
Tinghuai Ma a,b,*, Xiafei Suo ${ }^{\mathrm{a}}$, Jinjuan Zhou ${ }^{\mathrm{a}}$, Meili Tang ${ }^{\mathrm{c}}$, Donghai Guan ${ }^{\mathrm{d}}$, Yuan Tian ${ }^{\mathrm{e}}$, \\ Abdullah Al-Dhelaan ${ }^{\mathrm{e}}$, Mznah Al-Rodhaan ${ }^{\mathrm{e}}$ \\ ${ }^{a}$ School of Computer $\mathcal{E}$ Software, Nanjing University of information science $\mathcal{E}$ Technology, Jiangsu, Nanjing 210-044, China \\ ${ }^{b}$ Jiangsu Engineering Center of Network Monitoring, Nanjing University of information science $\mathcal{E}$ Technology, Nanjing 210-044,China \\ ${ }^{c}$ School of Public Administration, Nanjing University of Information Science $\mathcal{E}$ Technology,Nanjing 210044, China \\ ${ }^{d}$ college of computer science $\mathcal{E}$ technology, Nanjing university of Aeronautics $\mathcal{E}$ Astronautics, Nanjing, 210016, China \\ ${ }^{e}$ Computer Science Department, College of Computer and Information Science, King Saud University, Riyadh 11362, Saudi Arabia
}

\begin{abstract}
Recommender systems play an important role in our daily life and are becoming popular tools for users to find what they are really interested in. Matrix factorization methods, which are popular recommendation methods, have gained high attention these years. With the rapid growth of the Internet, lots of information has been created, like social network information, tags and so on. Along with these, a few matrix factorization approaches have been proposed which incorporate the personalized information of users or items. However, except for ratings, most of the matrix factorization models have utilized only one kind of information to understand users' interests. Considering the sparsity of information, in this paper, we try to investigate the combination of different information, like tags and genres, to reveal users' interests accurately. With regard to the generalization of genres, a constraint is added when genres are utilized to find users' similar "soulmates". In addition, item regularizer is also considered based on latent semantic indexing (LSI) method with the item tags. Our experiments are conducted on two real datasets: Movielens dataset and Douban dataset. The experimental results demonstrate that the combination of tags and genres is really helpful to reveal users' interests.
\end{abstract}

Keywords: Recommender systems, matrix factorization, tags, genres, LSI

\section{Introduction}

With the rapid development of the web, people are overwhelmed in a large amount of information. Explosive growth of information boosts more accurate recommendation techniques to be developed to instruct the users' decision-making process effectively. Generally, two types of recommender systems have been investigated: Memorybased [1,2] and Model-based [3,4] recommenders. As we know that, Memory-based recommenders predict user's rating on a particular item based on his/her rating history. Model-based recommenders are a little different from Memory-based methods, which learn some parameters when training a model. And they use the optimal model with the learned parameters to provide recommendations. The advantages of Model-based approaches are that they are fast and extensible, which means they could integrate many cases into one method or multiple kinds of information can be integrated into one model.

\footnotetext{
${ }^{*}$ Corresponding author: thma@ nuist.edu.cn
} 
Recently, with the appearance of diverse information, matrix factorization is used in recommend system[5]. The researchers integrate the personalized information into matrix factorization methods, aiming to utilize different information to relieve the sparsity of ratings and finally improve the recommendation quality[6-14]. Almost all of them are on the basis of rating matrix of users or items. They try to learn a latent user feature matrix and an item feature matrix by factorizing the whole rating matrix. Apart from this, many constraints are added in matrix factorization methods to optimize these models. For example, the researchers think that in the real life, users often get some recommendations from the people that they have similar interests to some degree $[9,10,15-17]$. The other people ask suggestion from their friends or the people they trust or people who they have some interactions with. Except for ratings, the data have been utilized in these models usually includes social network information [8-9, 15, 17-20], tags on items [5, 12, 14, 21-23] or item genres [24-27]. And all of these matrix factorization approaches have obtained good recommendation results.

However, except for ratings, we notice that most researchers have utilized user feature matrix and item feature matrix to optimize the models. And we have to acknowledge that, in reality, almost all information suffer sparsity problem [28]. In [10], except the factorization of rating matrix, only users trust friends favors are considered. While in [19], only tags are utilized to find the similar neighbors of users and items. About the item genres, Zhang et al. [13] have taken advantage of genres of items which users has rated to cluster users into groups and try to make users in the same group while they have the similar tastes. The advantage of the above researches is that all of them have reflected the effectiveness of the three kinds of information in modeling users' interests. However, due to the sparsity of information, we think more useful information should be considered to model users' interests more accurately and realistically.

So, in this paper, we focus on investigating the effectiveness of the matrix factorization method based on the combination of two kinds of information: tags and item genres. That is, we factorize the rating matrix to learn user latent feature matrix and item latent feature matrix. Moreover, we add extra regularization on users and items to optimize our model based on tags and genres information. In detail, we think target users are similar with their "soulmates" for who may have the same tagging behaviors or like the same type of films. These "soulmates" are not necessarily the social friends of target users. The connection of them may just because they have the same behaviors on websites. We find "soulmates" of target users based on both tags and genres which reflect users' interests in different aspects and we believe that an appropriate mode of combination of these two kinds of information may complement each other for the problem of sparsity. Meanwhile, we find similar items for target items through LSI method [25, 29] by tag information.

The remainder of this paper is organized as follows. In section 2, we provide an overview of matrix factorization approaches in recommender systems, as well as the information they have utilized. Section 3 presents the problem definition and preliminaries of matrix factorization. The details of our work are given in section 4 and the results of the empirical analysis are presented in section 5. In the last section, we conclude our work and present some future works.

\section{Related works}

Matrix factorization method is a model-based method which has achieved good performance in recommendations. We know that the Singular Value Decomposition (SVD) [30, 31] is a well-known matrix factorization technique. Traditionally, SVD [30] was used to factorize the user-item rating matrix which contains lots of missing values in it. While recently, many works $[6,9,10,14,23]$ focus on factorizing the observed ratings and adding regularizer in SVD to avoid overfitting. Probabilistic Matrix Factorization (PMF) [11] is a extend version of SVD from the point of probability. And most of matrix factorization approaches are proposed based on SVD or PMF. Here, we review the existing methods from two main aspects according to the information they used. One is the social network information, the other is tag information.

Firstly, we review some matrix factorization approaches which incorporate social network information $[9,10$, 15-20, 32-34]. SoRec [9] is proposed that a persons social network will affect personal behaviors on the Web. The social network matrix is factorized as well as the rating matrix. In order to model trust-aware recommender systems more realistically, STE [10] was proposed in which the users tastes and their trusted friends favors are fused together. The results show that STE outperforms PMF as well as SoRec. Based on the above two models, Ma et al. [17] proposed a general method, in which two general social regularizations are given: average-based regularization and 
individual-based regularization. The method can be easily extended to incorporate other contextual information. Later, considering the STE model does not handle trust propagation, Jamali and Ester [15] proposed SocialMF which incorporates trust propagation in matrix factorization and think that user $u$ 's feature vectors director neighbors affect the feature vector of user $u$ instead of the ratings of user $u$. Yang et al. [33] integrated twofold sparse information, the ratings and the social trusted network among users, into the matrix factorization technique, aiming to make users' reciprocal influence on their own opinions more reasonably. A slightly different from the above works which using social friendship or trusted relationship of users, Yuan et al. [34] take membership into consideration and focus on the analysis of factorization vs. regularization. And Ma et al. [32] use of users' commenting action in probabilistic matrix factorization. Apart from the works on public datasets, Shangguan et al. [35] applied matrix factorization method with social relationships in the book recommendation in douban website while Zhang et al. [36] and Bao et al.[37] have applied matrix factorization methods with social network information to Sina-weibo. Lin et al. [38, 39] investigated the problem of personalized news recommendation and proposed PRemiSE, a novel personalized news recommendation framework via implicit social experts.

The second popular information is tags. Recently, tags have received high attention in matrix factorization $[5,12$, 14, 21-23]. Zhen et al. [14] proposed TagiCoFi, in which user similarities are defined based on the tag information to regularize the matrix factorization procedure. Zhou et al. [23] proposed a factor analysis approach called TagRec by utilizing users' tags and ratings. In TagRec, user-tag tagging matrix and item-tag tagging matrix are factorized along with user-item rating matrix. Later, a model named NHPMF was proposed by Wu et al. [21]. In NHPMF, tag information is utilized to select neighbors of each user and each item. The intuition behind the model is that similar users (items) will have similar latent features. The experimental results show that NHPMF outperforms TagiCoFi and TagRec. Shi et al. [12] factorize the user-item matrix, in which contains general movie ratings and other contextual movie similarity matrices to integrate contextual information in the recommendation. The information that they focus on are movie mood tags and movie plot keywords. Different from other works related to social recommendation, Zhao et al. [22] merged topic mining and social trust analysis techniques into recommender systems for finding topics from the tags of the items and estimating the topic-specific social trust. They proposed a probabilistic matrix factorization algorithm by utilizing the estimated topic-specific social trust relations.

Apart from social network information and tags, item genres are also important to users [13, 24, 26, 27]. In real life, we usually like to watch a film which has the similar types or genres we like before. The genres are very convenient and helpful for us to make a decision. Zhang et al. [13] proposed a matrix factorization approach with the user clustering regularization. They use K-means algorithm to cluster users based on genres of items which users have rated and they think users have similar tastes in the same group. Manzato [26] proposes a recommender algorithm that is based on a factorized matrix composed of user preferences associated to the movies genres/categories. The advantage of using such user-genre matrix factorization model is that it requires less computational resources, as the matrix will be less sparse and at lower dimension. Nie et al. [27] proposed a novel method to measure users' preference on movie genres and a matrix factorization framework was introduced for genre preference regularization.

From the researches above, we can see that all the three kinds of information have been used to optimize the matrix factorization model. That means all of them could reflect users interests to some degree. However, except for ratings, most of matrix factorization models utilize only one kind of information to model users' interests. Moreover, in reality, apart from ratings, social network information and tags may have the sparsity problem. And from the above researches, we find that, in NHPMF model [21], only tags are utilized to find users' similar neighbors and in [13], only genres are used to find users who have similar tastes with target user. Considering the limitation of single information, like sparsity or something else, we try to merge tags and genres to reveal more accurate and realistic interests of users.

\section{Problem definition and preliminaries}

In recommender systems, users' ratings on items are usually used to learn users' preferences on items. Due to the sparsity of the ratings, the performances of the methods which only utilize rating information are limited. With the development of Web 2.0 applications, tagging technique has become a popular way for users to add personalized tags on web resources. Such tagging information may help us to understand the users' interests on items semantically. And an increasing number of methods $[14,21,23]$ get good recommendations when using tag information. To the best of our knowledge, not everyone likes to add personalized tags on items. Different people have different behaviors. That is, someone may rate a lot of items but tag just one item, while some people rate little but tag a lot. For people 
who tag little, his little historical tag information may not help understand his preferences significantly. This shows the sparsity of tag information, which is just like the ratings. As we know that, the content of items, to some degree, indicates some extra information of items, such as the categories, characteristics and so on. Among the content of items, genre information, which are considered as classes and special characteristics of items, is particular and been assigned by movie experts and directors. To alleviate the sparsity of ratings and tag information, in this paper, we try to merge genre information into tags for getting more accurate interests of users.

In recommender systems, we have $m$ users and $n$ items. Users ratings on items can be denoted as an $m^{*} n$ matrix $R$ where $R_{i j}(i=1,2, \ldots, m ; j=1,2, \ldots, n)$ represents the rating of the user $i$ for the item $j$. In some scenario, genre information may be available in the websites and through the items which user has rated on, we may get the personal genres that the user has involved. For example, movie $j$ has genres of "action", "comedy" and "fantasy"; movie $h$ has "action", "drama" and "comedy". If user $i$ has rated only the two movies, then we can define the genre vector of user $i: g_{\text {user }_{i}}=\{$ "action" : 2, "comedy" : 2, "fantasy" : 1, "drama" : 1\}. Similarly, with the development of Web2.0, in most systems, users are allowed to add tags to their interested items. If user $i$ has given tags "action" and "Oscar" to movie $j$ as well as "action" and "Ang Lee" to movie $h$, then we define the tag vector of user $i$ as $t_{\text {user }_{i}}=\{$ "action" $: 2$, "Oscar" : 1, "Ang Lee" $: 1\}$. The item tag vectors are defined just the same way of users.

In this paper, given ratings, tags and item genres, we employ matrix factorization method to characterize the users and items on latent factor space and predict the users' rating on items by the users' and items' latent feature vectors. The latent user and item feature matrices are denoted as $U \in \mathbb{R}^{l * m}$ and $V \in \mathbb{R}^{l * n}$, respectively. The dimension of the latent factor space $l$ satisfies $l \ll m, n$. The column vectors $u_{i}$ and $v_{j}$ with the dimensionality of $l$ represent the user-specific and item-specific latent feature vectors, respectively. In section 3.1, we will give a brief review of the low-rank matrix factorization, based on which, we propose our matrix factorization method.

\subsection{Low-rank matrix factorization}

The premise behind the low-dimensional factor model is that only a small number of factors play an important role [17]. In recommender systems, a low-rank matrix factorization approach tends to approximate rating matrix $R$ by multiplying $l$-rank factors:

$$
R \approx U^{T} V
$$

where $U \in \mathbb{R}^{l * m}$ and $V \in \mathbb{R}^{l * n}$ are latent user feature matrix and item feature matrix, respectively. Usually, the SVD method is utilized to approximate a rating matrix $R$ by minimizing:

$$
\frac{1}{2}\left\|R-U^{T} V\right\|_{F}^{2}
$$

where $\|.\|_{F}^{2}$ denotes the Frobenius norm. For the reason of sparsity of the ratings, a large number of missing values may be contained in the rating matrix $R$. So, instead of approximating the rating matrix $R$, we factorize the observed ratings in matrix $R$. And we redefine the objective function $E_{S V D}$ as:

$$
E_{S V D}=\min _{U, V} \frac{1}{2} \sum_{i=1}^{m} \sum_{j=1}^{n} I_{i j}\left(R_{i j}-u_{i}^{T} v_{j}\right)^{2}
$$

where $I_{i j}$ is an indicator function which is equal to 1 if user $i$ has rated item $j$ and equal to 0 otherwise. In order to avoid overfitting, two regularizers are added into the above objective function as:

$$
\begin{aligned}
E_{S V D}= & \min _{U, V} \frac{1}{2} \sum_{i=1}^{m} \sum_{j=1}^{n} I_{i j}\left(R_{i j}-u_{i}{ }^{T} v_{j}\right)^{2} \\
& +\frac{\lambda_{U}}{2}\|U\|_{F}^{2}+\frac{\lambda_{V}}{2}\|V\|_{F}^{2}
\end{aligned}
$$

where $\lambda_{U}, \lambda_{V}>0$. Another interpretation of this low-rank matrix factorization method from the point of probability is detailed in [11], which is named as probabilistic matrix factorization (PMF). 


\section{Proposed matrix factorization method}

In this section, we present a detail introduction of the proposed matrix factorization algorithm. In our algorithm, two regularizers are incorporated into the low-rank matrix factorization method introduced in section 3.1: the user regularizer and the item regularizer. Considering the sparsity of ratings and tags, we try to utilize both movie genres and tags to model a more accurate user regularizer. At the same time, item regularizer is taken into consideration in which latent semantic indexing method (LSI) [29] is used to find the top similar items of an item with item tag information.

\subsection{The user regularizer}

In reality, most people like to seek advice or get references from people who are similar with him/her. For example, user $i$ wants to buy a T-shirt from an online store, maybe she wishes to get some advice from user $k$ who has bought a T-shirt from the same online store before. Similarly, before watching a film, you may check the reviews of other people who have watched the film and then make a decision. Usually, people are very easy to be influenced silently by people who have the same interests with them. In most cases, we may expect to get references from all people who have similar behaviors or similar interests with us, but not being limited to our social friends. Therefore, in our method, we define "soulmates" from the point of global view. That is, we try to find all similar users of user $i$ and make a recommendation for user $i$ according to his/her top $N$ similar users.

Every user has his/her own taste and at the same time, every user may be influenced by his/her trusted friends[10]. So in our paper, we hold that users have their own interests as well as being influenced by their similar "soulmates" as measured by users' tag information and the genres of items. In [10], a weight parameter $\alpha$ is added to merge the influences of both user's own taste and their trusted friends:

$$
u_{i}^{T} v_{j}=\alpha u_{i}^{T} v_{j}+(1-\alpha) \sum_{k \in \mathcal{T}(i)} S_{i k} u_{k}^{T} v_{j}
$$

Eq. (5) is equivalent to the Eq. (6) as follows:

$$
u_{i}=\alpha u_{i}+(1-\alpha) \sum_{k \in \mathcal{T}(i)} S_{i k} u_{k}
$$

where parameter $\alpha$ controls how much users trust themselves or their trusted friends. $\mathcal{T}(i)$ is the set of friends that user $i$ trusts. $S_{i k}$ denotes how much user $i$ trusts the user $k$.

In our paper, we incorporate Eq. (6) into our matrix factorization and define the user regularizer $r_{u}$ as follows:

$$
\begin{aligned}
r_{u} & =\frac{\lambda_{\text {user }}}{2} \sum_{i=1}^{m}\left\|u_{i}-\left(\alpha u_{i}+(1-\alpha) \sum_{k \in \mathcal{T}(i)} S_{i k} u_{k}\right)\right\|^{2} \\
& =\frac{\lambda_{\text {user }}}{2} \sum_{i=1}^{m}(1-\alpha)^{2}\left\|u_{i}-\sum_{k \in \mathcal{T}(i)} S_{i k} u_{k}\right\|^{2}
\end{aligned}
$$

where $\lambda_{\text {user }}>0$ is a regular parameter to control the user regularization. $\alpha$ controls how much users depend on themselves or their similar "soulmates". $\mathcal{T}(i)$ is the set of "soulmates" of user $i . S_{i k}$ denotes the similarity between user $i$ and user $k$. Before giving the definition of $S_{i k}$, let us describe a phenomenon first. For the reason of the sparsity of ratings and tags, only one kind of information may not reflect users' interests globally and accurately. Although several researches [14, 21, 23] have achieved good results by matrix factorization methods with utilizing tag information, which is proved to be good at representing users interests, we still think the recommendation can be better. Let us imagine that, in real life, jack is not willing to tag movies or he always forgets to tag some movies. But interestingly, he is always used to rate movies since he thinks ratings express his opinions to those movies and maybe more intuitive for reference. Sometimes, he may give completely different ratings for two movies with the same genres. For example, he gives 5 stars for one movie and 1 star for another one just for a bad scene of this movie. From the point of ratings, we usually think low rating means dislike. But from another perspective of genres, we think jack likes both of the two movies for most movies he watched have similar genres with these two movies. So, we 
believe that movie genres users have involved, to some extent, they may help to reflect users' interests powerfully. Thus, in our user regularizer, we take both tags and genres into consideration, and define similarity of user $i$ and user $k$ as follows:

$$
S_{i k}=\left\{\begin{array}{l}
\beta * T_{i k}+(1-\beta) * G_{i k}, \text { if user } i \text { and user } k \text { have more than five tags } \\
(1-\beta) * G_{i k}, \text { otherwise. }
\end{array}\right.
$$

where $\beta$ is a parameter which controls the importance of tag similarity and genre similarity between users. $T_{i k}$ represents the similarity of user $i$ and user $k$ based on their tag vectors, while $G_{i k}$ denotes the similarity of user $i$ and user $k$ based on the genre vectors of them. Here, we think it is occasional if user $i$ has only one tag, and at the same time, we do not think less than five tags may help to find the users interests significantly. Therefore, if one of the two users or both have less than five tags, we define the tag similarity between the two users as zero. And in this situation, the similarity of two users only comes from the genre similarity. Before we compute the genre similarity and tag similarity of users, we first normalize the corresponding vectors and Vector Space Similarity (VSS) is employed to obtain both $T_{i k}$ and $G_{i k}$.

Actually, we think genres are more broader while tags are more specific. So if two users like different genres of movies, they may have totally different interests. But if they have different tags, their interests may have a small difference. Therefore, when using genres to express the preferences of users, some constraints must be made in $G_{i k}$. Before making the constraints, we first give a simple example of the genre similarity. There are three users: user $a$, user $b$, user $c$ and their genre vectors are as follows:

$$
\begin{aligned}
& g_{\text {user }}=\{" \text { action": 10, "comedy": 5, "horror": } 3\} \\
& g_{\text {user }_{b}}=\{\text { "action": 10, "comedy": 5, "fantasy": } 3\} \\
& g_{\text {user }_{c}}=\{" \text { action" : 5, "comedy": 5, "fantasy": 5\} }
\end{aligned}
$$

we assume that user $b$ hates horrible movies. On the surface, we consider user $b$ is more similar to user $c$ than user $a$. However, through the VSS of genre vectors, we get the conclusion that user $b$ is more similar to user $a$ than user $c$. The genre similarity between user $b$ and user $a$ is 0.9328 while user $b$ and user $c$ is 0.8977 . In order to get the real relationship between the three users, we redefine the genre similarity between two users as follows:

$$
G_{i k}=\left\{\begin{array}{l}
V S S\left(\text { user }_{i}, \text { user }_{k}\right), \text { if user } i \text { and user } k \text { have exactly the same genres } \\
0, \text { otherwise. }
\end{array}\right.
$$

Using Eq. (9), we may get new genre similarities of above three users. With the constraint, we find that the genre similarity of user $a$ and user $b$ will be zero for they have different genres: "horror" and "fantasy". Meanwhile, the genre similarity of user $b$ and user $c$ is 0.8977 . The new genre similarities show that user $b$ is more similar with user $c$ and it is consistent with the reality.

\subsection{The item regularizer}

Like users, items have their own "personalized" content. For example, a movie may have genres, directors, location, tags and so forth. In recommender systems, our aim is to recommend users with their possibly interested items. If we have obtained the users interests, the next thing we need to do is to match the most similar items with users' interests. Therefore, how to model the items based on their content is very important for recommender systems. No doubt that more accurate the item model, better the recommendation results. In our paper, we try to utilize tags items which have got from users to represent each item. Here, we do not consider the genres of items. For sake of model complexity, we only try the combination of tags and genres in user regularizer.

In our matrix factorization method, an effective item regularizer is needed to make better recommendations. Tags of items are used to represent each item. Similar with user's tag vector, an item tag vector is composed of tags that different users have tagged on it. Considering the free-form nature of tagging, one tag may have multi senses and multiple tags may have the same sense [40]. So, in order to avoid the problems above, we use LSI method instead of $\mathrm{TF}^{*}$ IDF to deal with the item tag vectors. In the item regularizer, LSI method is utilized to obtain the topic distribution of each item based on item tag vectors. LSI is a popular linear algebraic indexing method to produce low dimensional 
representations by word co-occurrence [29]. It takes advantage of implicit semantic structure in order to improve the detection of relevant documents. Given a specific topic number, LSI aims to find the best dimensional subspace approximation to the original documents space. In our paper, we take items as documents and tags of items as words in documents. Giving the items tag vectors, topic distributions of each item can be got by LSI and then the similarity between items could be easily obtained by items' topic distribution vectors. In order to improve the accuracy of the recommendation, we ignore items which have less than five tags in item regularizer. Compared to other methods, like TF*IDF, we expect that LSI is more competitive in the issues of synonymy and polysemy.

In our matrix factorization method, we define the item regularizer $r_{v}$ like follows:

$$
r_{v}=\frac{\lambda_{\text {item }}}{2} \sum_{j=1}^{n} \sum_{h \in F(j)} F_{j h}\left\|v_{j}-v_{h}\right\|^{2}
$$

where $\lambda_{\text {item }}>0$ is a regular parameter to control the item regularization. $F_{j h}$ is the similarity between item $j$ and item $h$ based on their topic distributions. $F(j)$ denotes the set of top $N$ similar items of item $j$.

\subsection{The MF_gener_tag_weight method}

After given the user regularizer and item regularizer, we propose our matrix factorization method. Our method, named MF_genre_tag_weight, integrates the above two regularizers into the low-rank matrix factorization method. The objective function of our method is as follows:

$$
E=E_{S V D}+r_{u}+r_{v}
$$

substitute the $E_{S V D}, r_{u}, r_{v}$ with Eq.(4), Eq.(7), Eg.(10) separately, we can get:

$$
\begin{aligned}
E= & \min _{U, V} \frac{1}{2} \sum_{i=1}^{m} \sum_{j=1}^{n} I_{i j}\left(R_{i j}-u_{i}^{T} v_{j}\right)^{2}+\frac{\lambda_{U}}{2}\|U\|_{F}^{2}+\frac{\lambda_{V}}{2}\|V\|_{F}^{2} \\
& +\frac{\lambda_{\text {user }}}{2} \sum_{i=1}^{m}(1-\alpha)^{2}\left\|u_{i}-\sum_{k \in \mathcal{T}(i)} S_{i k} u_{k}\right\|^{2} \\
& +\frac{\lambda_{\text {item }}}{2} \sum_{j=1}^{n} \sum_{h \in F(j)} F_{j h}\left\|v_{j}-v_{h}\right\|^{2}
\end{aligned}
$$

A local minimum of the objective function given by Eq. (12) can be found by performing gradient descent in feature vectors $u_{i}$ and $v_{j}$ :

$$
\begin{aligned}
\frac{\partial E}{\partial u_{i}}= & \sum_{j=1}^{n} I_{i j}\left(u_{i}^{T} v_{j}-R_{i j}\right) v_{j}+\lambda_{U} u_{i} \\
& +\lambda_{\text {user }}(1-\alpha)^{2}\left(u_{i}-\sum_{k \in \mathcal{T}(i)} S_{i k} u_{k}\right) \\
& -\lambda_{\text {user }} \sum_{g \in \mathcal{T} \backslash(i)} S_{g i}(1-\alpha)^{2}\left(u_{g}-\sum_{k \in \mathcal{T}(g)} S_{g k} u_{k}\right) \\
\frac{\partial E}{\partial v_{j}} & =\sum_{i=1}^{m} I_{i j}\left(u_{i}^{T} v_{j}-R_{i j}\right) u_{i}+\lambda_{V} v_{j} \\
& +\lambda_{\text {item }} \sum_{h \in F(j)} F_{j h}\left(v_{j}-v_{h}\right) \\
& +\lambda_{\text {item }} \sum_{g \in F \backslash(j)} F_{g j}\left(v_{j}-v_{g}\right)
\end{aligned}
$$



$j$.

where $\mathcal{T} \backslash(i)$ is the set of user $i$ 's top $N$ similar users and $F \backslash(j)$ represents the set of the top $N$ similar items of item

\section{Experiments}

In this section, we conduct several experiments to compare the recommendation result of our matrix factorization method with other state-of-the-art recommendation methods.

\subsection{Datasets}

For the utilization of genre information, we evaluate our methods on two real world datasets: Movielens [41] and Douban [21].

Movielens dataset, published by the GroupLens research group at University of Minnesota, is one of the most popular datasets used in the recommender systems. It is released in the framework of the $2^{\text {nd }}$ International Workshop on Information Heterogeneity and Fusion in Recommender Systems (HetRec 2011) [41]. It contains 2113 users, 10197 movies (items), 855,598 ratings and 13,222 tags in the dataset. Additionally, there are 20 movie genres and 20,809 movie genre assignments.

Another dataset is Douban. We got the initial Douban dataset from [21] and there are 1,500,000 records about the ratings and tags users have given to movies. We first remove the records which have no ratings and tags and then $1,161,171$ records are left. In the remaining records, we get 5,186 unique users and 39,501 items with 1,041,801 rating and 1,253,310 tag assignments. Then, we select users or items which have both ratings and tags. At the same time, in order to improve the recommendation accuracy, we filter out the users who have less than 20 ratings. In the end, there are 2,992 users and 31,613 items left with 866,099 ratings and 1,217,681 tag assignments. However, there is no genre information in the Douban dataset. In order to test our methods in Douban dataset, we crawl the corresponding movie genres of 31,613 items (movies in douban) from douban website and there are 41 unique genres. For the sake of time saving, we only select the first 1000 unique users in user-item-ratings file with 291,457 rating records. The corresponding items are 23,347 and 427,798 tag assignments. There are still 41 unique genres, but with 42,904 genre assignments. More statistics of our test datasets after preprocess can be seen in Table 1.

Table 1: More statistics of Movielens and Douban datasets

\begin{tabular}{lrr}
\hline Statistics & Movielens & Douban \\
\hline \#user & 2,113 & 1,000 \\
\#item & 10,197 & 23,347 \\
\#rating & 855,598 & 291,457 \\
\#tag & 13,222 & 20,864 \\
\#tag assignment & 47,957 & 427,798 \\
\#average tags per user & 22.70 & 427.80 \\
\#average tags per item & 8.12 & 18.32 \\
\#average rating per user & 404.92 & 291.46 \\
\#average rating per item & 84.64 & 12.48 \\
\#genre & 20 & 41 \\
\#genre assignment & 20,809 & 42,904 \\
\hline
\end{tabular}

We can see that in our two datasets, Movielens dataset has more ratings while Douban dataset has more tag assignments. The average tags per user or item in Douban dataset is larger than those in Movielens dataset while the average rating per user or item in Douban dataset is less than those in Movielens dataset. In the point of genres, Douban dataset has more unique genres as well as genre assignments.

For the both datasets, in experiments, we randomly select $70 \%, 80 \%, 90 \%$ of data for training, and the rest for testing. The random selection is executed 5 times independently. The average of 5 independent results is taken as the final result. 


\subsection{Metrics}

In this paper, two popular metrics are adopted to measure the recommendation quality in our experiments: the Mean Absolute Error (MAE) and the Root Mean Square Error (RMSE). The metrics MAE is defined as:

$$
M A E=\frac{\sum_{i, j}\left|R_{i j}-\hat{R}_{i j}\right|}{T}
$$

where $R_{i j}$ denotes the observed rating user $i$ has given to item $j . \hat{R}_{i j}$ denotes the predict rating of user $i$ on item $j$. $T$ represents the total number of tested ratings.

The metrics RMSE is defined as:

$$
R M S E=\sqrt{\frac{\sum_{i, j}\left(R_{i j}-\hat{R}_{i j}\right)^{2}}{T}}
$$

From the above definitions, we can see that a smaller MAE or RMSE value indicates higher recommendation accuracy.

\subsection{Parameters decision}

From the objective function Eq.(12), there need decide some parameters such as $\alpha, \lambda_{\text {item }}, \lambda_{\text {item }}, n$, and $\beta$ in Eq.(8). We find the optimized parameters through experiments.

\subsubsection{Impact of parameter $\alpha$}

In our paper, the parameter $\alpha$ in Eq. (6) controls how much users depend on themselves or their similar "soulmates" when make a decision. The parameter indicates that some connections may exist between the tastes of users and their "soulmates". If $\alpha=1$, the influence of the users"soulmates" will not be considered and only users own tastes are used. If $\alpha=0$, the users own tastes will be ignored and only users "soulmates" tastes are employed to make recommendations. Except for the above two extreme cases, we consider both users own tastes and the tastes of their similar users. We expect both of the information could contribute to the final recommendations.

For deciding the $\alpha$, we set the default value $\lambda_{\text {user }}=30, \lambda_{\text {item }}=5, n=5, \beta=0.5, \lambda_{U}=0.1, \lambda_{V}=0.1$. Then we change the $\alpha$ from 0 to 1 with step 0.1 .

Figure 1 shows the impacts of $\alpha$ on MAE and RMSE in Movielens dataset and Douban dataset, separately. It can be seen in Movielens dataset, the value of $\alpha$ impacts the recommendation results significantly. When $\alpha$ is larger than 0.4 , the values of MAE and RMSE increase quickly, especially when $\alpha=1$, the worst performance is achieved. When $\alpha$ is set from 0 to 0.4 , slight decline is shown on both MAE and RMSE. In Douban dataset, along with the changes of $\alpha$, the whole trends on MAE and RMSE are relatively steady. However, we still could find that both of MAE and RMSE decline when $\alpha$ is from 0 to 0.4 and an obvious increase is got when $\alpha$ is from 0.4 to 1 . From the trends on MAE and RMSE in both datasets, we also find that the impact of $\alpha$ on Movielens dataset is larger than that on Douban dataset.

Finally, we set $\alpha=0.4$ in our experiments as optimized value in both datasets.

\subsubsection{Impact of parameters $\lambda_{\text {user }}$ and $\lambda_{\text {item }}$}

The parameters $\lambda_{\text {user }}$ and $\lambda_{\text {item }}$ play important roles in our proposed approach. They control the strength of user regularizer and item regularizer, respectively. If both $\lambda_{\text {user }}$ and $\lambda_{\text {item }}$ are set to 0 , our method degrades to the basic matrix factorization method which only employs the observed rating information. The very small values of $\lambda_{\text {user }}$ and $\lambda_{\text {item }}$ mean that we mainly mind the observed ratings for recommendation and ignore the influences or connections of similar users and items. Oppositely, the very large value of $\lambda_{\text {user }}$ and $\lambda_{\text {item }}$ mean that we mainly depend on the similar soulmates of users or the similar items of target items for recommendation. Meanwhile, some other cases are also possible, for example, $\lambda_{\text {user }}$ is very large as $\lambda_{\text {item }}$ is very small, or otherwise. From the above all, we need to find the optimal combination of $\lambda_{\text {user }}$ and $\lambda_{\text {item }}$ in order to get the best recommendations.

Due to the large range of possible values for $\lambda_{\text {user }}$ and $\lambda_{\text {item }}$, we narrow the range in advance according to the experiments results. Finally, we test $\lambda_{\text {user }}$ in $1,25,30,35,40,80$ and $\lambda_{\text {item }}$ in $0.01,0.1,1,5,10,35$ in Movielens 


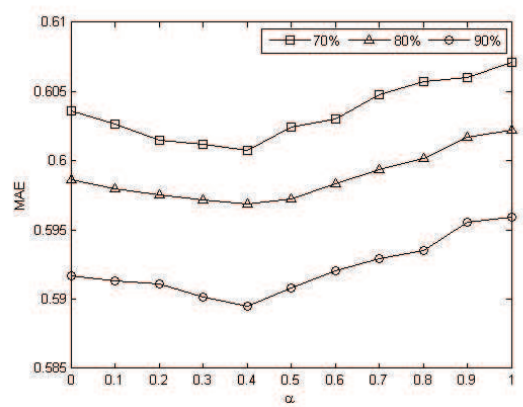

(a) Movielens (MAE)

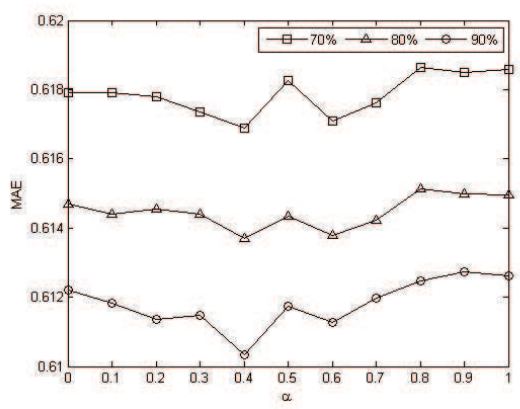

(c) Douban (MAE)

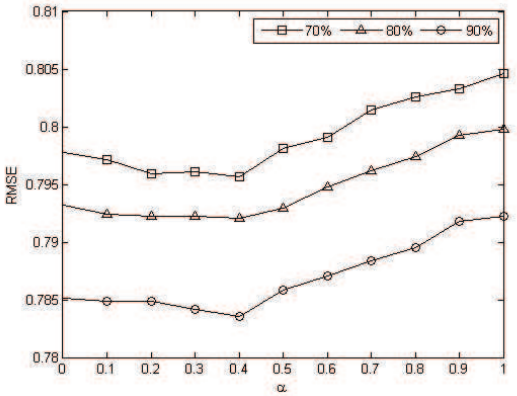

(b) Movielens (RMSE)

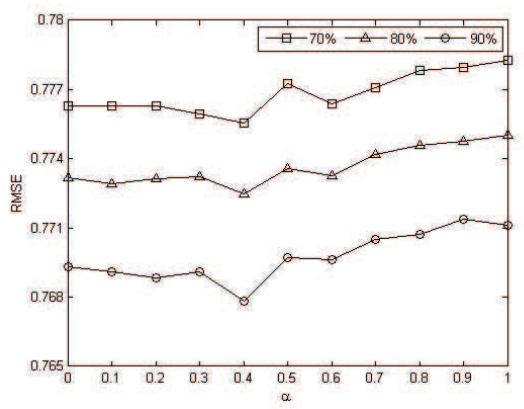

(d) Douban (RMSE)

Figure 1: Impact of Parameter $\alpha$

dataset and 5, 25, 30, 35, 40, 50 of $\lambda_{\text {user }}$ and $0.01,0.1,1,5,10,30$ of $\lambda_{\text {item }}$ in Douban dataset. Since the aim is to find the optimal combination, we test each combination of $\lambda_{\text {user }}$ and $\lambda_{\text {item }}$.

Here, in Movielens dataset, we set $\alpha=0.4$ which is got in section 5.3.1 as a optimal value, and set $n=5, \beta=0.5$, $\lambda_{U}=0.1, \lambda_{V}=0.1$ as default values. While in experiments, to test the impact of $\lambda_{\text {user }}$, we choose $\lambda_{\text {user }}$ from $1,25,30$, $35,40,80$ and fix the $\lambda_{\text {item }}=1$. In order to test the impact of $\lambda_{\text {item }}$, we fix the $\lambda_{\text {user }}=35$ while $\lambda_{\text {item }}$ is from $0.01,0.1$, $1,5,10,35$.

Similarly, in Douban dataset, set $\lambda_{\text {item }}=5$ when test $\lambda_{\text {user }}$ and set $\lambda_{\text {user }}=30$ when $\lambda_{\text {item }}$ is test. Figure 2 and Figure 3 show the impact of $\lambda_{\text {user }}$ and $\lambda_{\text {item }}$ in Movielens and Douban dataset separately.

From Figure 2, we see that best performance is achieved while $\lambda_{\text {user }}$ is around 35. When $\lambda_{\text {user }}$ is set to a small value, like 1, the MAE and RMSE increase dramatically. Moreover, in our parameter combinations, two special combinations $\lambda_{\text {user }}=\lambda_{\text {item }}=1$ and $\lambda_{\text {user }}=\lambda_{\text {item }}=35$ are also test. The corresponding results in Figure 2 show that both the two combinations get the worst performances in our method, which, to some degree, means $\lambda_{\text {user }}$ and $\lambda_{\text {item }}$ do not share the same trend in our method in Movielens dataset. The different results of $\lambda_{\text {user }}$ and $\lambda_{\text {item }}$ indicate that we should pay more attention on users influences or connections, and at the same time, the influences of similar items should not be ignored, either.

In our method, we set $\lambda_{\text {user }}=35$ and $\lambda_{\text {item }}=1$ in Movielens dataset.

Figure 3 shows the impact of $\lambda_{\text {user }}$ and $\lambda_{\text {item }}$ in Douban dataset. From Fiure 3(a) and Figure 3(b), we see that our method gets the best performance when $\lambda_{\text {user }}$ is 30. The impact of $\lambda_{\text {item }}$ on MAE and RMSE is shown in Figure 3(c) and Figure 3(d). When $\lambda_{\text {item }}=5$, the best result is achieved. The trend of $\lambda_{\text {item }}$ in Douban dataset is different from that in Movielens dataset, and large values of $\lambda_{\text {item }}$ tends to get poor results. At the same time, in Douban dataset, we also test two special combinations $\lambda_{\text {user }}=\lambda_{\text {item }}=5$ and $\lambda_{\text {user }}=\lambda_{\text {item }}=30$. The results show that both of the combinations get poor results. The results of special parameter combinations in our test in both datasets show that the impact of $\lambda_{\text {user }}$ does not share the same trend as the impact of $\lambda_{\text {item }}$ in our method.

Finally, we set $\lambda_{\text {user }}=30$ and $\lambda_{\text {item }}=5$ in Douban dataset. 


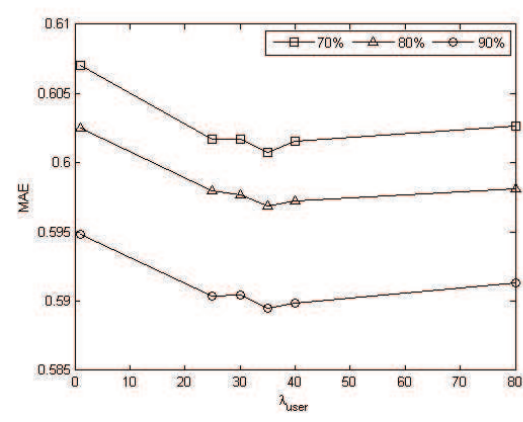

(a) $\lambda_{\text {user }}$ (MAE)

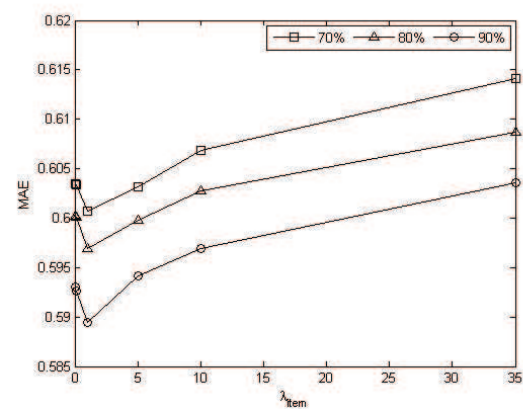

(c) $\lambda_{\text {item }}(\mathrm{MAE})$

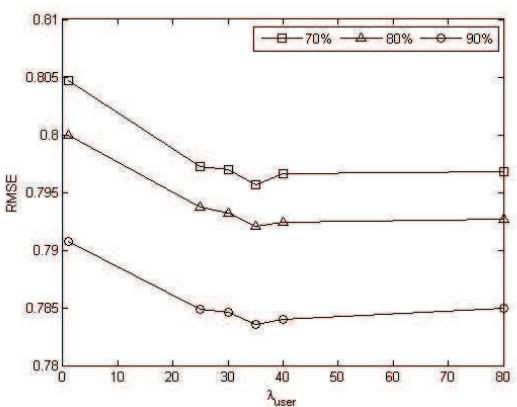

(b) $\lambda_{\text {user }}$ (RMSE)

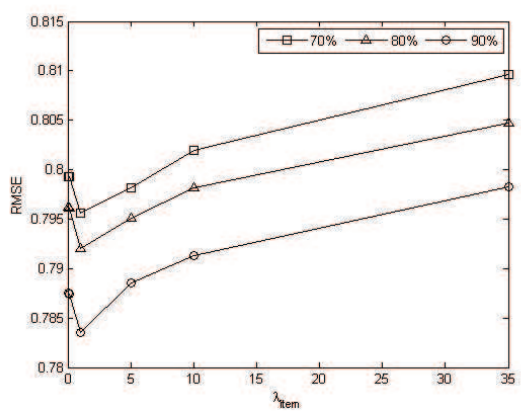

(d) $\lambda_{\text {item }}$ (RMSE)

Figure 2: Impact of $\lambda_{\text {user }}$ and $\lambda_{\text {item }}$ in Movielens dataset

\subsubsection{Impact of parameter $\beta$}

In our proposed method MF_genre_tag_weight, $\beta$ plays an important role in the user reuglarizer. It is a weight parameter that controls the proportion of users tag similarity and genre similarity. In the extreme case, $\beta=0$ means that users top similar "soulmates" are got only based on their genre information and $\beta=1$ means only tag information are considered when find users top similar "soulmates". In many cases, we do not choose the extreme values, since we believe that both of tags and genres have their weakness and they need to complement each other. So, we tune $\beta$ from 0 to 1 and analyze how the changes of $\beta$ can affect the recommendation results.

For choosing the best $\beta$, in experiments, in Movielens dataset, we set $\alpha=0.4, \lambda_{\text {user }}=35, \lambda_{\text {item }}=1$ which is got in above sections, and set $n=5, \lambda_{U}=0.1, \lambda_{V}=0.1$ as default values. In Douban dataset, the $\lambda_{\text {user }}=30, \lambda_{\text {item }}=5$ while other parameters are same.

Figure 4 shows the impacts of $\beta$ on MAE and RMSE in two datasets of our proposed method. We notice that, in Movielens dataset, as $\beta$ increases, the MAE and RMSE values decreases at first, but when $\beta$ goes beyond a certain threshold, the MAE and RMSE values increase with further increase of $\beta$. However, in Douban dataset, the trend is not as explicit as that in Movielens dataset. The trend in Douban Dataset is relatively flat with the varying $\beta$. Even though, we still can find a lowest point in the trendline.

From the Figure 4, we can see that when $\beta$ is set to 0.9 , our method gets the best performance in both datasets, which demonstrates that both tags and genres are helpful to model the interests of users.

\subsubsection{Impact of parameter $n$}

In the item regularizer, LSI method was used to get the item topic distribution vector on the basis of item tag vectors. In LSI, $n$ is a parameter which represents the dimension of the latent semantic space. That is, LSI aims to find a $n$ dimensional subspace approximation to the given documents space. Different number of $n$ may lead to totally different approximations. A larger value of $n$ means that the latent subspace of approximation is close to the original one which indicates that LSI method does not reach the goal of dimensionality reduction and noise reduction. Here, 


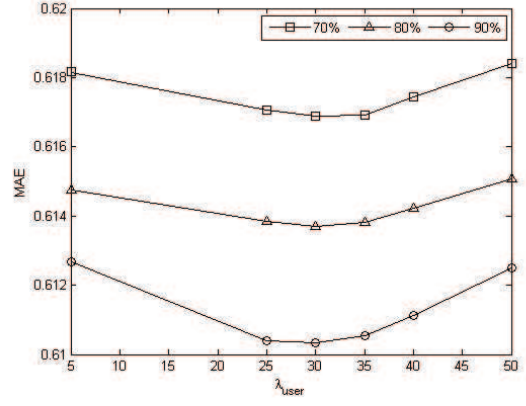

(a) $\lambda_{\text {user }}$ (MAE)

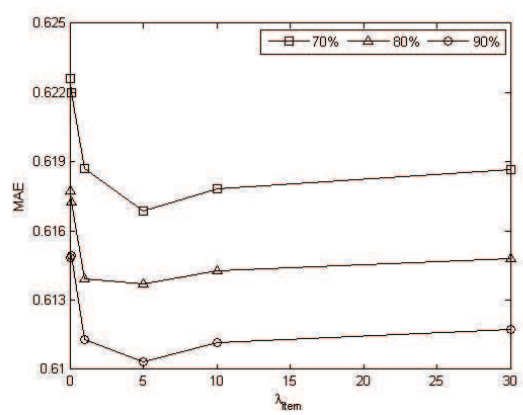

(c) $\lambda_{\text {item }}(\mathrm{MAE})$

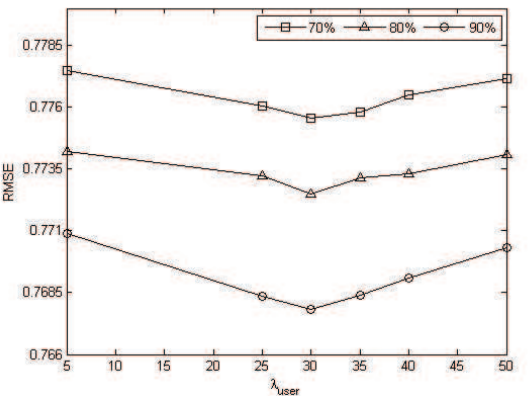

(b) $\lambda_{\text {user }}$ (RMSE)

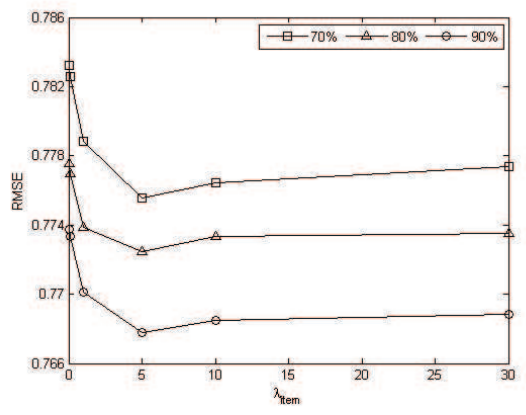

(d) $\lambda_{\text {item }}(\mathrm{RMSE})$

Figure 3: Impact of $\lambda_{\text {user }}$ and $\lambda_{\text {item }}$ in Douban dataset

we conduct an experimental analysis with different value of $n$ as 5,10,15,20 and other parameters is set as optimized value as above discussion. The experimental results are shown in Table 2 and Table 3. We also show the histogram of results in Figure 5 and Figure 6.

Table 2: topic number $n$ in Movielens dataset

\begin{tabular}{cccccc}
\hline training & metrics & 5 & 10 & 15 & 20 \\
\hline \multirow{2}{*}{$70 \%$} & MAE & $\mathbf{0 . 6 0 0 7}$ & 0.6007 & 0.6012 & 0.6012 \\
& RMSE & $\mathbf{0 . 7 9 5 6}$ & 0.7957 & 0.7963 & 0.7964 \\
\hline \multirow{2}{*}{$80 \%$} & MAE & 0.5969 & $\mathbf{0 . 5 9 6 6}$ & 0.5968 & 0.5967 \\
& RMSE & 0.7921 & $\mathbf{0 . 7 9 1 9}$ & 0.7919 & 0.7921 \\
\hline \multirow{2}{*}{$90 \%$} & MAE & $\mathbf{0 . 5 8 9 5}$ & 0.5896 & 0.5906 & 0.5901 \\
& RMSE & $\mathbf{0 . 7 8 3 5}$ & 0.7839 & 0.7851 & 0.7841 \\
\hline
\end{tabular}

Table 3: topic number $n$ in Douban dataset

\begin{tabular}{cccccc}
\hline training & metrics & 5 & 10 & 15 & 20 \\
\hline \multirow{2}{*}{$70 \%$} & MAE & 0.6169 & 0.6175 & $\mathbf{0 . 6 1 6 6}$ & 0.6191 \\
& RMSE & 0.7755 & 0.7770 & $\mathbf{0 . 7 7 5 2}$ & 0.7782 \\
\hline \multirow{2}{*}{$80 \%$} & MAE & $\mathbf{0 . 6 1 3 7}$ & 0.6138 & 0.6143 & 0.6144 \\
& RMSE & $\mathbf{0 . 7 7 2 5}$ & 0.7732 & 0.7734 & 0.7735 \\
\hline \multirow{2}{*}{$90 \%$} & MAE & $\mathbf{0 . 6 1 0 3}$ & 0.6112 & 0.6107 & 0.6110 \\
& RMSE & $\mathbf{0 . 7 6 7 8}$ & 0.7692 & 0.7684 & 0.7688 \\
\hline
\end{tabular}




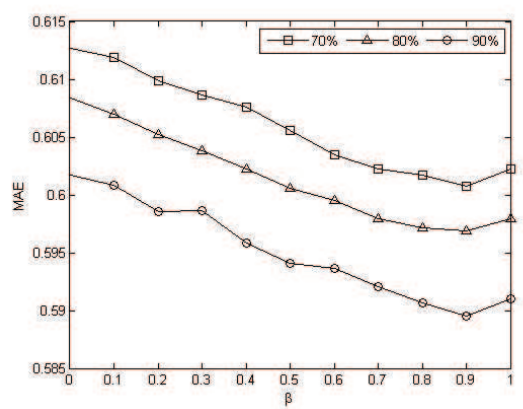

(a) Movielens (MAE)

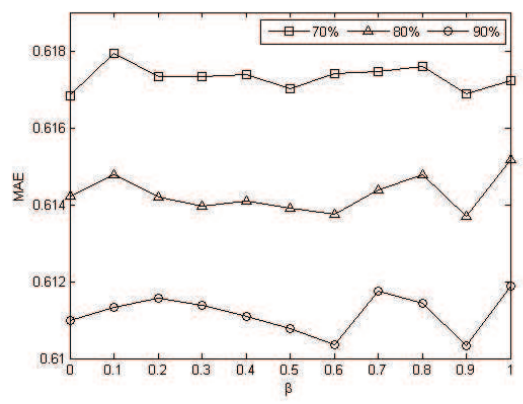

(c) Douban (MAE)

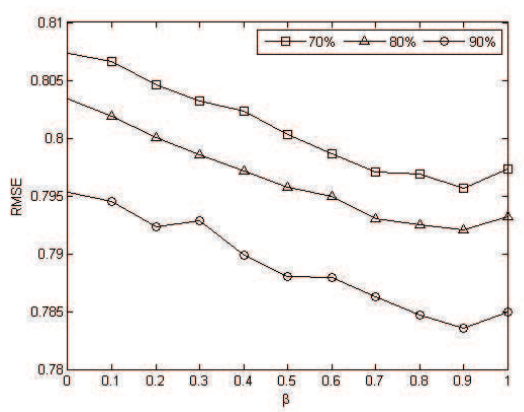

(b) Movielens (RMSE)

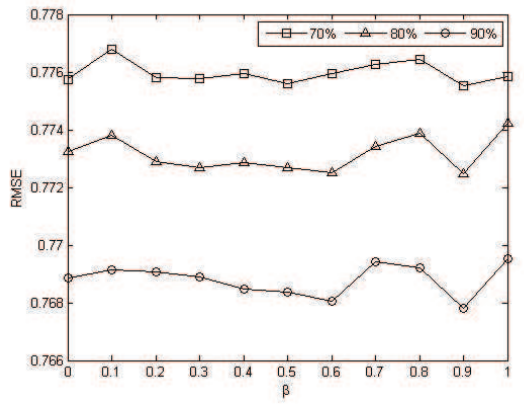

(d) Douban (RMSE)

Figure 4: Impact of Parameter $\beta$

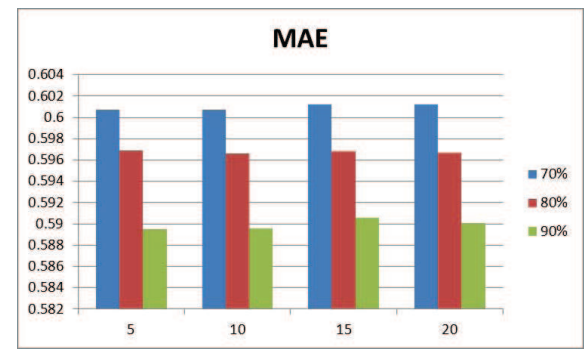

(a) Movielens (MAE)

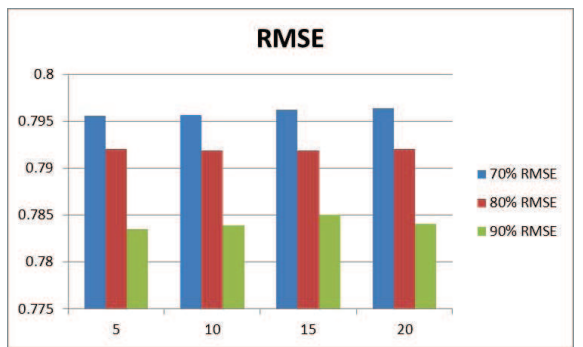

(b) Movielens (RMSE)

Figure 5: topic number $n$ in Movielens dataset 
Shown in Table 2, in the settings of $70 \%$ of data for training and $90 \%$ of data for training, best performances are achieved as $n$ is set to 5, while in the setting of $80 \%$ of data for training, the best result is obtained with $n=10$. Similar situation is happened in Douban dataset in Table 3. Although there is no value of $n$ gets best in all the three settings of both datasets, there still exists a parameter value which is relatively better.

Finally, we choose $n=5$ for both datasets for the reason that $n=5$ gets best in most cases in the two datasets.

\subsubsection{Impact of the constraint in genre similarity}

In the user regularizer, a constraint is made when genre vectors are used to obtain users genre similarity. Considering the generalization of genres, we do not think they can be used in the same way with tags which are relatively individual and narrow. To verify our guess, we do some changes in MF_genre_tag_weight and produce a new method, named MF_NOgenre_tag_weight in which genre similarity is obtained just the same way of that in tag similarity. That is, both the genre similarity and tag similarity are acquired through the VSS of corresponding vectors.

Table 4: the performance of different limitations of genres

\begin{tabular}{ccccc}
\hline dataset & training & metrics & MF_genre_tag_weight & MF_NOgenre_tag_weight \\
\hline \multirow{5}{*}{ Movielens } & \multirow{2}{*}{$70 \%$} & MAE & $\mathbf{0 . 6 0 0 7}$ & 0.6016 \\
& \multirow{2}{*}{$80 \%$} & RMSE & $\mathbf{0 . 7 9 5 6}$ & 0.7969 \\
\cline { 2 - 5 } & \multirow{2}{*}{$90 \%$} & MAE & $\mathbf{0 . 5 9 6 9}$ & 0.5970 \\
& \multirow{2}{*}{ MASE } & $\mathbf{0 . 7 9 2 1}$ & 0.7923 \\
\hline \multirow{5}{*}{ Douban } & RMSE & $\mathbf{0 . 5 8 9 5}$ & 0.5912 \\
& \multirow{2}{*}{$70 \%$} & MAE & $\mathbf{0 . 6 1 6 3 5}$ & 0.7855 \\
\cline { 2 - 5 } & \multirow{2}{*}{$80 \%$} & RMSE & $\mathbf{0 . 7 7 5 5}$ & 0.6175 \\
& \multirow{2}{*}{9} & MAE & $\mathbf{0 . 6 1 3 7}$ & 0.7764 \\
\hline \multirow{2}{*}{$90 \%$} & MAE & $\mathbf{0 . 7 7 2 5}$ & 0.6138 \\
& & RMSE & $\mathbf{0 . 6 1 0 3}$ & 0.7726 \\
\hline
\end{tabular}

Table 4 gives the results of the above two methods and we can see that in both datasets, MF_genre_tag_weight is superior to MF_NOgenre_tag_weight, even though it is only a slight improvement. The results suggest that the constraint in genre similarity is meaningful and our idea is reasonable.

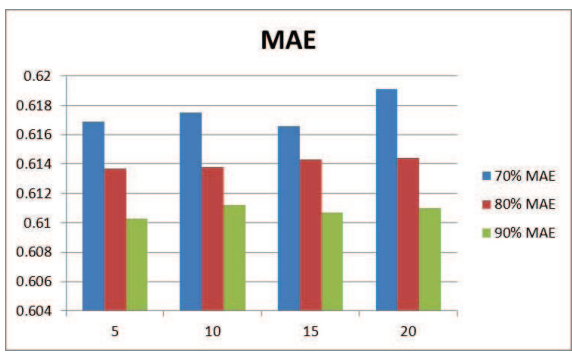

(a) Douban (MAE)

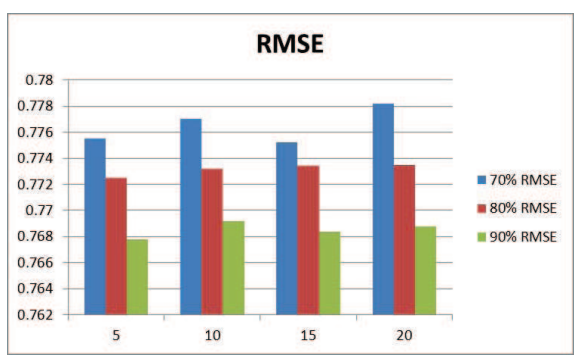

(b) Douban (RMSE)

Figure 6: topic number $n$ in Douban dataset

\subsection{Results}

\subsubsection{Comparison}

In both datasets, we randomly select $70 \%, 80 \%, 90 \%$ of ratings for training, and the rest for testing. The random selection was carried out 5 times independently. And the last result comes from the average of five independent experiments. 
To evaluate the performance of our method we consider four comparison partners. We use the basic matrix factorization method from Salakhutdinov[11], and implement the three methods based on the code of basic matrix factorization method by ourselves.

- PMF: This method is a baseline matrix factorization approach proposed in [11].

- MF_cluster: This model is proposed in [25], which integrating the user clustering regularization term into the standard MF. It clusters the users with movie genres users have involved. No item regularization is considered.

- NHPMF: This model is proposed in [19], which utilizing the tagging data to select neighbors of each user and each item and then adding unique Gaussian distributions on each users (items) latent feature vector in MF to ensure similar users (items) will have similar latent features.

- MF_tag: This method is a simple version of our proposed method MF_genre_tag_weight. The only difference between MF_tag and MF_genre_tag_weight is user similarity and item similarity. In MF_tag, we use TF*IDF to compute the similarity between users or items based on user tag vectors or item tag vectors.

In our experiments, we name our proposed model as MF_genre_tag_weight. In MF_genre_tag_weight, user regularizer and item regularizer are considered. The user similarities in user ragularizer are got by Eq. (8) where the tag similarity and the genre similarity are smoothed by the parameter $\beta$. The item similarities in item regularizer are obtained through the LSI method on the basis of item tag vectors. In the above four comparison partners, MF_tag model also integrates the user regularizer in Eq. (7) and item regularizer in Eq. (10). The difference between the MF_tag and MF_genre_tag_weight is the way users or items similarity are obtained. In MF_tag, TF*IDF is used to get the user similarity and item similarity without consideration of genres or LSI method. Meanwhile, the same point between the MF_tag and the NHPMF is that both of them get the user or item similarity through TF*IDF based on user or item tag vectors. And the difference is that they have different matrix factorization framework.

For verifying our methods, we set parameters as optimal values as we discussed in above section. But different algorithm has different parameter configuration.

- we set $\lambda_{U}=\lambda_{V}=0.1$ for all methods except NHPMF. In NHPMF, if there is no other external data source to extract the neighbors of users and items, the model degenerates to the basic low-rank matrix factorization. So, the parameters $\lambda_{U}$ and $\lambda_{V}$ in NHPMF are a little different from those in basic low-rank matrix factorization. In the above two datasets, we set $\lambda_{U}=\lambda_{V}=5$ for NHPMF and it is the best of what we found in parameter tuning.

- In MF_cluster, the user clustering regularization parameter is set to 0.001 in both datasets. And we test different values of clustering number $k$ in two datasets and finally we set $k$ to 5 in Movielens dataset and 6 in Douban dataset.

- For MF_tag and MF_genre_tag_weight, we set $\lambda_{\text {user }}=35$ and $\lambda_{\text {item }}=1$ in Movielens dataset while $\lambda_{\text {user }}=30$ and $\lambda_{\text {item }}=5$ in Douban dataset. We set $\alpha$ in MF_tag and MF_genre_tag_weight to 0.4 in both datasets and $N=5$ in both user regularizer and item regularizer. Meanwhile, we find that $\beta=0.9$ and $n=5$ are the best combination in both datasets.

- overall, for both Movielens dataset and Douban dataset, the dimensionality of the latent feature space in our experiments is set to 10 . In gradient descending, the step size for all methods is empirically set to 0.01 . The maximum of the step is 1000 for after 1000 steps, all methods are stable.

The whole experimental results are shown in Table 5. As we can see from the results, our method consistently outperforms other approaches in both datasets. MF_tag, which has the same matrix factorization framework with MF_genre_tag_weight, also outperforms the other three comparison methods. The performances of MF_genre_tag_weight and MF_tag indicate that our matrix factorization framework is superior to the other three in both datasets. The advantage is especially shown in the comparison of MF_tag and NHPMF. We know that both MF_tag and NHPMF have the same user and item similarity through TF*IDF on the basis of user or item tag vectors and the only difference is the matrix factorization framework. Meanwhile, MF_genre_tag_weight is the best of the five methods, which demonstrates that the user regularizer and item regularizer in our method indeed brought great improvement in recommendation 
Table 5: Performance Comparisons in two datasets

\begin{tabular}{|c|c|c|c|c|c|c|c|}
\hline dataset & training & metrics & PMF[11] & MF_cluster[25] & NHPMF[19] & MF_tag & MF_genre_tag_weight \\
\hline \multirow{6}{*}{ Movielens } & \multirow{2}{*}{$70 \%$} & MAE & 0.6207 & 0.6108 & 0.6050 & 0.6040 & 0.6007 \\
\hline & & RMSE & 0.8154 & 0.8091 & 0.8002 & 0.7992 & 0.7956 \\
\hline & \multirow{2}{*}{$80 \%$} & MAE & 0.6173 & 0.6033 & 0.6002 & 0.5987 & 0.5969 \\
\hline & & RMSE & 0.8124 & 0.8013 & 0.7957 & 0.7939 & 0.7921 \\
\hline & \multirow{2}{*}{$90 \%$} & MAE & 0.6133 & 0.5963 & 0.5942 & 0.5919 & 0.5895 \\
\hline & & RMSE & 0.8077 & 0.7930 & 0.7887 & 0.7863 & 0.7835 \\
\hline \multirow{6}{*}{ Douban } & \multirow{2}{*}{$70 \%$} & MAE & 0.6185 & 0.6305 & 0.6209 & 0.6173 & 0.6169 \\
\hline & & RMSE & 0.7769 & 0.7949 & 0.7808 & 0.7760 & 0.7755 \\
\hline & \multirow{2}{*}{$80 \%$} & MAE & 0.6153 & 0.6261 & 0.6170 & 0.6139 & 0.6137 \\
\hline & & RMSE & 0.7737 & 0.7905 & 0.7771 & 0.7732 & 0.7725 \\
\hline & \multirow{2}{*}{$90 \%$} & MAE & 0.6125 & 0.6223 & 0.6134 & 0.6105 & 0.6103 \\
\hline & & RMSE & 0.7698 & 0.7842 & 0.7720 & 0.7681 & 0.7678 \\
\hline
\end{tabular}

compared to other comparison partners. At the same time, with the consideration of genres, our method alleviates the sparsity of ratings and tags.

We also notice that in different dataset, different results are obtained by the five methods. We find that in both datasets, the improvement of MF_genre_tag_weight outperform NHPMF is stable in vary data environment. The NHPMF outperform MF_cluster becuse that MF_cluster only has user clustering regularizier while NHPMF has both user regularizer and item regularizer; or maybe MF_cluster utilizes only genres to find the users friends and get the user similarity with the ratings while NHPMF uses tags. Moreover, we notice that, in both datasets, MF_genre_tag_weight is always the best and MF_tag is the second.

In Movielens dataset, both NHPMF and MF_cluster outperform PMF while in Douban dataset, both of them are less superior than PMF. The bad performance of MF_cluster and NHPMF in Douban dataset may be caused by the nosie data in the dataset which we do not filter meaningless tags in datasets and just select the first 1000 users after simple pre-processing of the original datasets. The best performances of our method in both datasets demonstrate that our approach is stable and efficient.

\subsubsection{Statistical test}

As we consider two metrics to verify our results, the statistical analysis will consider these two groups performance measures, separately. With the results in table 5, we think it is multiple-problem analysis and the non-parametric statistical tests is adopt[23].

In statistical analysis, we want to check whether the results of the algorithms are rather significant for considering them in different jobs. As we proposed the MF_tag, MF_genre_tag_weight algorithms, which has lowest MAE and RMSE in the comparison will be studied carefully. The $p$-values on each comparison will be computed to determine the results MF_genre_tag_weight offers are better than any other algorithms.

Table 6 shows the results of applying Friedman's order to see whether there are global differences in the results. Given that the $p$-values of Friedman are lower than the level of significance considered $\alpha=0.05$, there are significant differences among the observed results in the MAE group and RMSE group.

From Table 7, the MF_genre_tag_weight algorithm outperforms the PMF[11], MF_cluster[25],NHPMF[19], MF_tag with a level of significance $\alpha=0.05$, because the all $p$-values of MF_genre_tag_weight vs. PMF[11], MF_genre_tag_weight vs. MF_cluster[25], MF_genre_tag_weight vs. NHPMF[19], MF_genre_tag_weight vs. MF_tag are lower than $\alpha$. Here we can declare that MF_genre_tag_weigh obtains a significantly better performance than PMF, MF_cluster, NHPMF, MF_tag algorithms.

\section{Conclusion and future works}

In this paper, we focus on investigating the combination of tags and genres and proposed a novel matrix factorization method which may alleviate the sparsity of single information, like ratings or tags. From the previous researches, 
Table 6: Ranking obtained through Friedman's test

\begin{tabular}{lll}
\hline \multirow{2}{*}{ Algorithm } & Ranking & \\
\cline { 2 - 3 } & MAE & RMSE \\
\hline PMF[11] & 3.83 & 4.00 \\
MF_cluster[25] & 4.50 & 4.50 \\
NHPMF[19] & 3.42 & 3.50 \\
MF_tag & 2.25 & 2.00 \\
MF_genre_tag_weight & 1.00 & 1.00 \\
Values in_ $\chi^{2}$ & 18.588 & 20.400 \\
$p$-value & 0.001 & 0.000 \\
\hline
\end{tabular}

Table 7: Ranking obtained through Friedman's test

\begin{tabular}{|c|c|c|c|c|c|c|}
\hline \multirow{2}{*}{$\begin{array}{l}\text { MF_genre_tag_weight } \\
\text { vs. }\end{array}$} & \multicolumn{3}{|l|}{ NR } & \multicolumn{3}{|l|}{ EM } \\
\hline & $\mathrm{R}+$ & $\mathrm{R}-$ & $p$-value & $\mathrm{R}+$ & $\mathrm{R}-$ & $p$-value \\
\hline$\overline{P M F[11]}$ & .00 & 3.50 & .027 & .00 & 3.50 & .028 \\
\hline MF_cluster[25] & .00 & 3.50 & .028 & .00 & 3.50 & .028 \\
\hline NHPMF[19] & .00 & 3.50 & .028 & .00 & 3.50 & .027 \\
\hline MF_tag & .00 & 3.50 & .028 & .00 & 3.50 & .028 \\
\hline
\end{tabular}

we find that almost all kinds of information could be utilized to find or reveal the interests of target users. However, except for ratings, in most cases, most kinds of information are used alone in matrix factorization approaches. Considering the sparsity of information, we try to merge tags and genres in an appropriate way in order to improve the accuracy of recommendations. In consideration of the generality of genres, a constraint is made when genres are utilized. Moreover, item regularizer is also considered in our method. With regard to the issues of synonymy and polysemy, LSI method was used to find similar items of target items on the basis of item tags. The experimental analysis on two real-world datasets shows that our approach has better performance than the compared partners.

In this paper, genres and tags are merged in an appropriate way to complement each other and a better recommendation result has been achieved. Actually, any kinds of information may be merged in their proper modes. The key point is that we need to know the strength and weakness of information and find appropriate ways to make up their defects. Meanwhile, in our method, no social network information was used because we think that we need to find users similar soulmates in global view, but not the social friends only. Maybe, in future, we will try to take social network information into consideration.

In our method, we pay more attention to find users interests and ignore the match of similar items. Although LSI method was used to find similar items for target item, there still have too much work for item regularizer. For the rich information of items, like ratings, tags, genres, directors, actors or actress, locations and so on, a more accurate and realistic model may be proposed in future. It may get amazing result along with the user regularizer. Meanwhile, we have to admit that parameter tuning is complex in our model. The advantage of this model is that a variety of personalized data is considered. The diversity of data makes a more precise recommendation. At the same time, the model has strong extensibility. In future, we could try to unify similar parameters or select test point at intervals. The shortage of this model is too many parameters make the tuning complex. We will decrease the complexity of parameter tuning in the future. 


\section{Acknowledgment}

This work was supported in part by National Science Foundation of China (No.61572259, 61173143), Special Public Sector Research Program of China (No. GYHY201506080), and was also supported by PAPD.

The authors extend their appreciation to the Deanship of Scientific Research at King Saud University for funding this work through research group no. RGP-VPP-264.

\section{References}

[1] G. Adomavicius and A. Tuzhilin. Toward the next generation of recommender systems: a survey of the state-of-the-art and possible extensions. IEEE Transactions on Knowledge and Data Engineering, 17(6), pp. 734-749, 2005.

[2] Karen H. L. Tso-Sutter, Leandro Balby Marinho, Lars Schmidt-Thieme, Tag-aware recommender systems by fusion of collaborative filtering algorithms, Proceedings of the 2008 ACM symposium on Applied computing, Fortaleza, Ceara, Brazil, pp.1995-1999, 2008.

[3] J. S. Breese, D. Heckerman, and C. Kadie, Empirical analysis of predictive algorithms for collaborative filtering, Proc. of the 14th Conference on Uncertainty in Artificial Intelligence, Madison, Wisconsin, pp. 43-52, 1998.

[4] X. Su, and T. M. Khoshgoftaar, A survey of collaborative filtering techniques, Advances in Artificial Intelligence, 2009, pp. 1-19, 2009.

[5] T. Bao, Y. Ge, E. Chen, H. Xiong, and J. Tian, Collaborative filtering with user ratings and tags, Proc. of the 1st International Workshop on Context Discovery and Data Mining, Beijing, China, pp. 1-5, 2012.

[6] Y. Koren, Factorization meets the neighborhood: a multifaceted collaborative filtering model, Proc. of the 14th ACM SIGKDD International Conference on Knowledge Discovery and Data Mining, Las Vegas, Nevada, USA, pp. 426-434, 2008.

[7] Bin Gu, Victor S. Sheng, Keng Yeow Tay, Walter Romano, and Shuo Li, Incremental Support Vector Learning for Ordinal Regression, IEEE Transactions on Neural Networks and Learning Systems, 26(7), pp.1403-16, 2015.

[8] Y. Koren, R. Bell, and C. Volinsky, Matrix Factorization Techniques for Recommender Systems, Computer, 42(8), pp. 30-37, 2009.

[9] H. Ma, H. Yang, M. R. Lyu, and I. King, SoRec: social recommendation using probabilistic matrix factorization, Proc. of the 17th ACM Conference on Information and Knowledge Management, Napa Valley, California, USA, pp. 931-940, 2008.

[10] H. Ma, I. King, and M. R. Lyu, Learning to recommend with social trust ensemble, Proc. of the 32nd International ACM SIGIR Conference on Research and Development in Information Retrieval, Boston, MA, USA, pp. 203-210, 2009.

[11] R. Salakhutdinov, and A. Mnih, Probabilistic matrix factorization, Advances in Neural Information Processing Systems, 20, pp. 1257-1264, 2008.

[12] Y. Shi, M. Larson, and A. Hanjalic, Mining contextual movie similarity with matrix factorization for context-aware recommendation, $A C M$ Trans. Intell. Syst. Technol., 4(1), pp. 1-19, 2013.

[13] C.-X. Zhang, Z.-K. Zhang, L. Yu, C. Liu, H. Liu, and X.-Y. Yan, Information filtering via collaborative user clustering modeling, Physica A: Statistical Mechanics and its Applications, 396, pp. 195-203, 2014

[14] Z.Zhang, L. Yu, K. Fang, Website-oriented recommendation based on heat spreading and tag-aware collaborative filtering, Physica A- Statical mechanics and its application, 399, pp.82-88, 2014.

[15] M. Jamali, and M. Ester, A matrix factorization technique with trust propagation for recommendation in social networks, Proc. of the 4th ACM Conference on Recommender Systems, Barcelona, Spain, pp. 135-142, 2010.

[16] M. Jamali, and M. Ester, A transitivity aware matrix factorization model for recommendation in social networks, Proc. of the 22nd International Joint Conference on Artificial Intelligence, Barcelona, Catalonia, Spain, pp. 2644-2649, 2011.

[17] H. Ma, D. Zhou, C. Liu, M. R. Lyu, and I. King, Recommender systems with social regularization, Proc. of the 4th ACM International Conference on Web Search and Data Mining, Hong Kong, China, pp. 287-296, 2011.

[18] J. Liu, C. Wu, and W. Liu, Bayesian Probabilistic Matrix Factorization with Social Relations and Item Contents for recommendation, Decision Support Systems, 55(3), pp. 838-850, 2013.

[19] H. Ma, I. King, and M. R. Lyu, Learning to recommend with explicit and implicit social relations, ACM Transactions on Intelligent Systems and Technology, 2(3), 2011.

[20] X. Yang, H. Steck, Y. Guo, and Y. Liu, On top-k recommendation using social networks, Proc. of the 6th ACM Conference on Recommender Systems, Dublin, Ireland, pp. 67-74, 2012.

[21] L. Wu, E. Chen, Q. Liu, L. Xu, T. Bao, and L. Zhang, Leveraging tagging for neighborhood-aware probabilistic matrix factorization, Proc. of the 21st ACM International Conference on Information and Knowledge Management, Maui, Hawaii, USA, pp. 1854-1858, 2012.

[22] T. Zhao, C. Li, M. Li, Q. Ding, and L. Li, Social recommendation incorporating topic mining and social trust analysis, Proc. of the 22nd ACM International Conference on Information and Knowledge Management, San Francisco, California, USA, pp. 1643-1648, 2013.

[23] T. C. Zhou, M. Hao, I. King, and M. R. Lyu, TagRec: Leveraging Tagging Wisdom for Recommendation, Proc. of the International Conference on Computational Science and Engineering, pp. 194-199, 2009.

[24] S.-M. Choi, S.-K. Ko, and Y.-S. Han, A movie recommendation algorithm based on genre correlations, Expert Systems with Applications, 39(9), pp. 8079-8085, 2012.

[25] C. Ding, T. Li, and W. Peng, On the equivalence between Non-negative Matrix Factorization and Probabilistic Latent Semantic Indexing, Computational Statistics $\mathcal{E}$ Data Analysis, 52(8), pp. 3913-3927, 2008.

[26] M. G. Manzato, Discovering latent factors from movies genres for enhanced recommendation, Proc. of the 6th ACM Conference on Recommender Systems, Dublin, Ireland, pp. 249-252, 2012.

[27] D. Nie, L. Hong, and T. Zhu, Movie Recommendation Using Unrated Data, Proc. of the 2013 12th International Conference on Machine Learning and Applications, pp. 344-347, 2013.

[28] J. Pinho Lucas, S. Segrera, and M. N. Moreno, Making use of associative classifiers in order to alleviate typical drawbacks in recommender systems, Expert Systems with Applications, 39(1), pp. 1273-1283, 2012. 
[29] W. Zhang, T. Yoshida, and X. Tang, A comparative study of TF*IDF, LSI and multi-words for text classification, Expert Systems with Applications, 38(3), pp. 2758-2765, 2011.

[30] B. Sarwar, G. Karypis, J. Konstan, and J. Riedl, Application of Dimensionality Reduction in Recommender Systems: A case study, WebKDD Workshop at the ACM SIGKKD, 2000.

[31] B. Sarwar, G. Karypis, J. Konstan, and J. Riedl, Incremental Singular Value Decomposition Algorithms for Highly Scalable Recommender Systems, Proc. of the 5th International Conference in Computers and Information Technology, 2002.

[32] D. Ma, D. Song, and L. Liao, Incorporating social actions into recommender systems, Proc. of the 14th International Conference on Web-Age Information Management, Beidaihe, China, pp. 698-704, 2013.

[33] Yinghua Lv, Tinghuai Ma, Meili Tang, Jie Cao, Yuan Tian, Abdullah Al-Dhelaan, Mznah Al-Rodhaan, An efficient and scalable density-based clustering algorithm for datasets with complex structures, Neurocomputing, 171, pp. 9-22, 2016

[34] Q. Yuan, L. Chen, and S. Zhao, Factorization vs. regularization: fusing heterogeneous social relationships in top-n recommendation, Proc. of the 5th ACM Conference on Recommender systems, Chicago, Illinois, USA, pp. 245-252, 2011.

[35] Q. Shangguan, L. Hu, J. Cao, and G. Xu, Book Recommendation Based on Joint Multi-relational Model, Proc. of the 2012 2nd International Conference on Cloud and Green Computing, pp. 523-530, 2012.

[36] Y. Zhang, W. Chen, and Z. Yin, Collaborative filtering with social regularization for TV program recommendation, Knowledge-Based Systems, 54, pp. 310-317, 2013.

[37] H. Bao, Q. Li, S. S. Liao, S. Song, and H. Gao, A new temporal and social PMF-based method to predict users' interests in micro-blogging, Decision Support Systems, 55(3), pp. 698-709, 2013.

[38] Tinghuai Ma, Huan Rong, Changhong Ying, Yuan Tian, Abdullah Al-Dhelaan, and Mznah Al-Rodhaan. Detect structural-connected communities based on BSCHEF in C-DBLP, Concurrency Computat.: Pract. Exper., 28(2), pp. 311-330, 2016

[39] C. Lin, R. Xie, X. Guan, L. Li, and T. Li, Personalized news recommendation via implicit social experts, Information Sciences, 254, pp. 1-18, 2014.

[40] J. Chen, S. Feng, and J. Liu, Topic sense induction from social tags based on non-negative matrix factorization, Information Sciences, 280, pp. 16-25, 2014.

[41] Tinghuai Ma, Jinjuan Zhou, Meili Tang, Yuan Tian, Abdullah AL-DHELAAN, Mznah AL-RODHAAN, and sungyoung Lee, Social network and tag sources based augmenting collaborative recommender system, IEICE transaction on Information and Systems, 98-D(4), pp.902-910, 2015. 